\title{
Einsatz von Recommender-Systemen zum Management von Unternehmensportalen
}

\author{
Helmuth Elsner, Jan Krämer \\ Institute of Information Systems and Management, \\ Karlsrube Institute of Technology (KIT) \\ \{Helmuth.Elsner|Jan.Kraemer\}@kit.edu
}

\section{Einleitung}

Zahlreiche Geschäftsmodelle im Internet basieren auf der Technologie von Webportalen. Eines der bekanntesten Beispiele ist das Internetportal Yahoo, welches eine Vielfalt von Diensten wie zum Beispiel Webmail, Wettervorhersagen und Nachrichtendienste anbietet. Die Stärke der Portaltechnologie ist es, den Zugriff auf eine Vielzahl von Diensten über einen singulären Einstiegspunkt anzubieten. Parallel zum stetigen Wachstum von Portalen im frei zugänglichen Internet wurde die Technologie erfolgreich für Zusammenarbeit zwischen und innerhalb von Unternehmen aufgenommen. Diese Unternehmensportale - im Englischen „Corporate Portals“ (Murray 1999; Dias 2001, S. 270) oder „Enterprise (Information) Portals" (Shilakes und Tylman 1998, S. 2; White 2000, S. 354-362) genannt - bieten dem Anwender eine Vielzahl von Möglichkeiten, die bisher nur partiell ausgenutzt werden. Die Einsatzfelder liegen zum einen im Bereich der Unternehmenskooperation, beispielsweise als Handelsplattform, wie sie im Automobilbereich in Form des Portals covisint (Arbin und Essler 2002, S. 279-292) existiert; zum anderen können sie innerhalb eines Unternehmens eingesetzt werden, um die Kommunikation mit und zwischen den Mitarbeitern zu vereinfachen (Knorr 2004). Bisher wurden Unternehmensportale besonders in Bezug auf ihre Fähigkeiten im Bereich des Wissensmanagements untersucht (Terra und Gorden, S. 1-419; Benbya et al. 2004, S. 201-22). Weiterführende Einsatzmöglichkeiten zielen auf die Integration verschiedener Anwendungen und das damit verbundene Potential, Geschäftsprozesse zu koordinieren und zu optimieren ab (Dias 2001, S. 274-279; Murray 1999), werden aber nicht weiter evaluiert. White spricht in diesem Zusammenhang von einer Entwicklung hin zu „Enterprise Application Suites“ (2003, S. 2). Die Herausforderungen die mit dieser Evolution von Unternehmensportalen verbunden sind, sollen in dieser Arbeit näher analysiert und Ansätze für das Management eines solchen Portals vorgestellt werden. 
Der vorliegende Artikel ist wie folgt gegliedert: Zunächst wird detailliert auf die Entwicklung von Unternehmensportalen hin zu komplexen Applikationsportalen mit integrierten Diensten zur Steuerung von Geschäftsprozessen eingegangen. Aufbauend auf dieser Analyse werden im dritten Abschnitt die Herausforderungen an das Management solcher Portale identifiziert. Hierbei wird einerseits der Aspekt des Wachstums in Bezug auf Anzahl und Komplexität der angebotenen Dienste als auch in Bezug auf die Erweiterung und Fluktuation der Nutzergruppe berücksichtigt. Um das Potential eines Portals voll ausnutzen zu können muss vorrangig sichergestellt werden, dass die Mitarbeiter die für sie relevanten Dienste kennen und nutzen. Gerade in dieser Herausforderung unterscheiden sich Unternehmensportale von öffentlich im Internet angebotenen. Schließlich wird ein Konzept für ein Recommender-System vorgestellt, welches personalisierte Empfehlungen für Dienste im Unternehmenskontext bereitstellen kann.

\section{Entwicklung von Unternehmensportalen}

Das Konzept von Unternehmensportalen wurde zuerst von Murray (1999) und Shilakes und Tylman (2000) vorgestellt. Beide beschreiben darin zwar primär das Potential in Bezug auf das Wissensmanagement von Unternehmen, aber gerade Murray hebt auch hervor, dass die Technologie weiter gehende Konzepte unterstützt. Die meisten der hierauf aufbauenden wissenschaftlichen Arbeiten beziehen sich vorrangig auf Wissensmanagement-Funktionalitäten. Diese umfassen in der Regel Suchfunktionalitäten nach Inhalt und META-Daten (Benbya et al. 2004, S. 210), Abonnements für Meldungen über E-Mail, RSS-Feeds oder Webservices (Dias 2001, S. 281) sowie Kollaborationsfunktionalitäten wie Dokumentenverwaltung oder Wikis (Eckerson 1999). Weiterhin wird in der Literatur auf die Möglichkeiten eingegangen, wie Inhalte personalisiert dargestellt werden können, um somit die Unternehmenskommunikation effizienter zu gestalten. Hervorzuheben ist hier insbesondere die Funktionalität Taxonomien zu erstellen mit deren Hilfe verwandte Elemente gruppiert dargestellt werden können (Aneja et al. 2000, S. 3-4; Detlor 2000, S. 93; Benbya et al. 2004, S. 206). Hinzu kommen die personalisierte Darstellung von Inhalten auf Grund von META-Daten oder Gruppenzuweisungen (Benbya et al. 2004, S. 211; Aneja et al. 2000, S. 4-5) und die Möglichkeit Empfehlungen über Inhalte abzugeben (Zanker et al. 2007, S. 273-276). Dias gibt in ihrer Arbeit eine ausführlichen Überblick über Literatur zu diesen Themen (2001). Zusätzlich beschreibt sie auch die Evolution von Unternehmensportalen und stellt dabei - mit Verweis auf die Vorarbeit von Eckerson (1999) - vier Generationen von Unternehmensportalen vor. Die vierte und letzte Generation, die Dias als „Spezialisierte Portale" bezeichnet, beschreibt das Portal als Unterstützungsfunktion für verschiedenste Unternehmensaufgaben. In dieser Generation wird das Portal mit Anwendungen des Unternehmens verknüpft, so dass Nutzer Transaktionen ausführen und Daten lesen und schreiben können (Dias 2001, S. 277). Hier zeichnet 
sich das Potential von Unternehmensportalen ab, dass bereits Murray beschrieben hatte. White bietet eine ähnliche Definition des vierten Levels. Er beschreibt ein Unternehmensportal als „Enterprise Application Suite“ deren Hauptzweck die Integration verschiedener Anwendungen und das Erstellen einer integrierten Geschäftsanwendung sei (2003, S. 2). Die Steuerung von Geschäftsprozessen ist natürlich eine Funktionalität, deren Ausprägung stark abhängig von der Art des jeweiligen Unternehmens ist. Allgemein betrachtet sind jedoch zwei Aspekte hervorzuheben, die auf diese Kategorie zutreffen.

Erstens können Business Intelligence Dienste angeboten werden, die in der Lage sind neue Information zu generieren, indem sie die bestehende Datenbasen aus den verschiedenen Quellen des Portals abgreifen, kombinieren und analysieren. Ebenso ist die Integration externer Datenquellen möglich, die über Application Interfaces (APIs) angebunden werden können (Eckerson 1999, S. 14). Erweitert können solche Dienste auch (halb-)automatisch Entscheidungen treffen und somit Prozessabläufe steuern. Als Beispiel sei die Kombination eines einfachen Wissensmanagement-Tools mit der Eingabemaske für eine integrierte Anwendung genannt. Ein intelligenter Prozess kann hier den Nutzer bei der Eingabe mit Vorschlägen unterstützen und in weiteren Schritten sogar die eingegebenen Werte mit historischen Daten vergleichen und validieren. Für den Nutzer liegt der Vorteil darin, dass er die zusätzlichen Informationen in der Anzeigemaske angezeigt bekommt, ohne aktiv nach ihnen suchen zu müssen und zudem mögliche Fehler bei der Eingabe durch den Vergleich mit Bestanddaten vermieden werden können.

Der zweite Aspekt sind automatische Reporting-Funktionalitäten bei der Steuerung von Geschäftsprozessen. Diese können entweder durch bestimmte Ereignisse ausgelöst werden oder auf einem Zeitplan basieren. Hier können Business Intelligence Dienste bei der Erstellung helfen (Benbya et al. 2004, S. 209) oder den Versand anstoßen. Das automatische Reporting nutzt die zuvor genannte Abonnementfunktionalität, um die Daten zum Nutzer zu liefern. Hervorzuheben ist die Option, die es ermöglicht, über automatisierte Berichte Fehlermeldungen oder Warnungen zu versenden. Auf diese Weise können Warnungen versendet werden wenn ein Prozess fehlerhaft abläuft oder wichtige Termine anstehen.

Die Unterstützung der unternehmensinternen Kommunikation und Prozessabläufe über ein Unternehmensportal hat im Vergleich zur Installation einzelner Anwendungen auf den Computern der Endanwender weiterhin den Vorteil, dass nur ein geringer Aufwand bei der Installation und Aktualisierung entsteht. Für den Zugang zum Portal benötigt man lediglich einen Webbrowser. Aktualisierungen des Portals können zentral vorgenommen werden und sind direkt verfügbar. Zusätzlich wird verhindert, dass mehrere Versionen parallel aktiv sind (Elsner und Vo 2007, S. 343-344).

Die Möglichkeiten, die ein Portal in Bezug auf die Unterstützung verschiedenster Unternehmensprozesse bieten kann, sind also sehr vielfältig und führen in der Folge zu einem stetigen Wachstum des Portals sowie in der Komplexität als auch in der Zahl der Dienste und Nutzer. Das Management des Portalwachstums und 
seiner Folgen stellt daher eine zentrale Herausforderung beim Einsatz von Unternehmensportalen der vierten Generation dar. Explizite Lösungsansätze zur Bewältigung dieser Problematik finden sich bisher nicht in der Literatur, die lediglich die Möglichkeiten des Portalkonzepts in Unternehmen hervorhebt.

Im Gegensatz zu Internetportalen verfolgen Unternehmensportale kein eigenes Geschäftsmodell, sondern unterstützen Unternehmen in der Verfolgung ihres Geschäftsmodells. Dieser fundamentale Unterschied führt auch zu anderen Anforderungen an das jeweilige Portalmanagement. Eine Gegenüberstellung der wichtigsten Unterschiede zwischen beiden Portaltypen ist durch Tabelle 1 gegeben.

Tabelle 1: Unterschiede zwischen Unternehmens- und Internetportalen

\begin{tabular}{l|ll}
\hline & Unternehmensportal & Internetportal \\
\hline Zweck & Unterstützt Geschäftsmodell & Ist Geschäftsmodell \\
Umgebung & Unternehmen/Intranet & Internet \\
Potentielle Nutzer & Angestellte & Internetgemeinschaft \\
Dienstkomplexität & Hoch & $\begin{array}{l}\text { Begrenzt durch Skalierbarkeit } \\
\text { und Verständlichkeit }\end{array}$ \\
& & FAQ, Hotline \\
Support & Training, Persönlich, Hotline, & \\
Zugriffskontrolle & Rollenbasiertes System & Einfache Authentifizierung \\
\hline
\end{tabular}

Der Hauptgrund für die Einführung eines Unternehmensportals ist es, die Produktivität der Mitarbeiter zu steigern und nicht einen möglichsten breiten Nutzerkreis durch attraktive Dienste anzusprechen. Im Gegensatz zu Internetportalen ist der Nutzerkreis im Unternehmen überschaubar und es kann von speziellen Voraussetzungen der Nutzer ausgegangen werden. Die verfügbaren Dienste in Unternehmensportalen können daher sehr spezialisiert und hochkomplex sein. Innerhalb eines Unternehmens ist es auch durchaus möglich, die Portaladministratoren direkt zu kontaktieren oder Nutzungsschulungen anzubieten. Ebenso ist die Gewährung von Zugriffsrechten bei Unternehmensportalen wesentlich vielschichtiger. Es werden in der Regel rollenbasierte Zugriffssysteme eingesetzt, um Nutzer von verschiedenen Abteilungen mit unterschiedlichen Aufgaben und Vertraulichkeitsstufen auf die für sie relevanten Dienste zuzulassen.

Die zuvor genannten Aspekte zeigen die im Allgemeinen deutlich erhöhte Nutzungskomplexität von Unternehmensportalen der vierten Generation im Vergleich zu Internetportalen auf. Die zentrale Herausforderung des Portalmanagements liegt darin, Mitarbeiter optimal bei der Nutzung des Portals zu unterstützen, wobei das stetige Wachstum besonders berücksichtigt werden muss. Einerseits sollen Mitarbeiter auf die ihnen bekannten Dienste weiter zugreifen können. Andererseits 
kann das Potenzial des Portals aber nur entfaltet werden, wenn andere relevante Dienste auch wahrgenommen werden und zu deren Nutzung angeregt wird.

In Bezug auf das Verhältnis zwischen Nutzer und Portal ist die Frage der Motivation hervorzuheben. Besucher eines Internetportals besuchen dieses aus eigenem Interesse, sei es um die Funktionalitäten zu erkunden, oder ein Produkt zu erwerben. Sie haben zudem meist die Möglichkeit, zwischen verschiedenen Anbietern zu wählen. Dieser intrinsischen Motivation steht die eher extrinsische Motivation der Nutzer von Unternehmensportalen gegenüber. Diese müssen das Portal nutzen, um ihre Arbeit zu erledigen. Das Interesse, das Portal zu erkunden wird hier in der Regel gering sein. Selbst wenn ein eigenständiges Interesse zum regelmäßigen Durchsuchen des Portals nach neuen und relevanten Diensten besteht, so wird dies sehr zeitaufwändig und somit ineffizient oder sogar auf Grund mangelnder Rechte unmöglich sein.

Aus diesem Vergleich leitet sich ab, dass das Managementkonzept für Unternehmensportale nicht einfach dem eingeschlagenen Pfad von Internetportalen folgen kann. Für den Betrieb eines erfolgreichen Unternehmensportals ist es notwendig, jedem Nutzer individuell die Vorteile des Portals für seine tägliche Arbeit darzulegen und ihn beim Auffinden neuer und relevanter Dienste effektiv zu unterstützen. In diesem Zusammenhang ist es hilfreich aus Sicht jedes Mitarbeiters individuell zwischen zwei Gruppen von Diensten zu unterscheiden: Die Gruppe der Muss-Dienste zeichnet sich durch jene Dienste aus, die der Mitarbeiter im Unternehmensportal nutzen muss, um einen speziellen Prozess ausführen zu können. Bei dieser Dienstgruppe kann also davon ausgegangen werden, dass sie dem jeweiligen Mitarbeiter bekannt sind. Andererseits kann es auch Prozesse geben, die ohne das Portal zwar erfolgreich ausgeführt werden können, unter Zuhilfenahme dessen aber zu einem besseren Ergebnis führen könnten. Solange ein Mitarbeiter aber auch ohne das Portal einen Prozess erledigen kann, wird er kaum motiviert sein, eigenständig nach Verbesserungsmöglichkeiten zu suchen. Diese Soll-Dienste gilt es daher individuell zu identifizieren und dem jeweiligen Mitarbeiter kenntlich zu machen, ohne dabei aufdringlich zu werden.

Die Problematik liegt zunächst in der individuellen Erkennung der SollDienste für jeden Mitarbeiter. Es ist offensichtlich, dass die ausführliche und regelmäßige Dokumentation aller Arbeitsprozesse eines Mitarbeiters im heutigen Unternehmensumfeld nicht praktikabel ist. Ausgehend von der Annahme, dass jeder Dienst jedoch zumindest einigen Nutzern bekannt ist (oder sich jeder Dienst in der Gruppe der Muss-Dienste einiger weniger Mitarbeiter befindet), besteht die Möglichkeit, das Nutzungsverhalten direkt durch das Portal analysieren zu lassen, um daraus Rückschlüsse auf entsprechende Soll-Dienste zu schließen. Die Ergebnisse dieser Analyse können in Empfehlungen führen, die das Portal seinen Nutzern personalisiert anbieten kann. Eben dies ist die Zielsetzung des RecommenderSystems, welches im folgenden Abschnitt genauer entwickelt wird. 


\section{Ein Recommender-System für Unternehmensportale}

Allgemein operiert ein Recommender-System auf einer Menge von Nutzern, $U$, und einer Menge von Objekten, $O$, eines Systems, $S=(U, O)$. Die Aufgabe des Recommender-Systems ist es nun, einem aktivem Nutzer $a \in U$ eine Empfehlung, $e \in O$, für weitere, bisher nicht genutzte Objekte zu geben ohne ihm dabei alle möglichen Objekte zu zeigen. Ziel ist es also, die dem Nutzer unbekannten Objekte so zu filtern, dass nur solche Objekte angezeigt werden, die potentiell relevant sind. Die individuelle Relevanz der Objekte wird hierbei durch den Vergleich von verschiedenen Nutzerprofilen abgeleitet: Sind die Profile zweiter Nutzer ähnlich, so wird auch davon ausgegangen, dass diese Nutzer die gleiche Dienstmenge nutzen sollten. Relevant sind dann insbesondere die Objekte, die nicht in der Schnittmenge der Dienste von ähnlichen Nutzern liegen.

Im Kontext von Unternehmensportalen werden spezielle Anforderungen an die Eigenschaften eines Recommender-Systems gestellt. Basierend auf den Ausführungen im vorherigen Abschnitt und den Praxiserfahrung der Autoren im Zusammenhang mit der Entwicklung und Betreuung eines Unternehmensportals der vierten Generation für eines der Dax30 Unternehmen, wird im Weiteren von folgenden Voraussetzungen ausgegangen:

- Das Portal bietet wenige hundert Dienste an und wird von einigen tausend Mitarbeitern genutzt

- Die zur Verfügung gestellten Dienste dienen als Schnittstelle zu Datenquellen und Anwendungen des Unternehmens. Es handelt sich also nicht um ein reines Wissensmanagementportal welches lediglich Dokumente anbietet.

- Die Nutzer können oder werden das Portal nicht eigenständig nach weiteren hilfreichen Diensten durchsuchen. Das Portal bietet jedoch zahlreiche Dienste an, die sie bei ihrer Arbeit unterstützen könnten.

- Über die Nutzer liegen Profildaten vor anhand derer sie grob gruppiert werden können.

- Jeder Mitarbeiter kann dem Recommender-System (implizit oder explizit) bekannt geben, welche Dienste er regelmäßig nutzt.

Im Vergleich mit Filmdatenbanken oder Buchversandportalen, in denen häufig Recommender-Systeme eingesetzt werden, ist die Zahl der Objekte und Nutzer im Unternehmenskontext relativ überschaubar. Der Fokus liegt zudem auf regelmäßig genutzten Diensten im Gegensatz zu Nachrichten oder Dokumenten, die meist nur einmalig aufgerufen werden. Somit wird die Zahl der relevanten Objekte pro Nutzer vergleichsweise klein sein. Die Aufgabe des Systems ist es daher möglichst alle relevanten Objekte zu identifizieren. Herlocker et al. (2004, S. 9-10) beschreiben diese Aufgabe als „Find all good items“. Im Bereich der RecommenderSysteme für personalisierte Empfehlungen stehen grundsätzlich zwei alternative Ansätze zur Wahl: Collaborative und Content-Based Filtering (Montaner et al. 2003, S. 298-299). Im Folgenden werden beide Ansätze auf ihre Verwendbarkeit für Unternehmensportale untersucht. 


\subsection{Content-Based Filtering}

Content-Based Filtering wird häufig in Nachrichtenportalen, Restaurantführern, Fernsehprogrammen oder Online-Shops eingesetzt. Das Konzept baut auf zwei Schritten auf: Im ersten werden Objekte des Systems durch Attribute mit festen Werten beschrieben. Im zweiten werden von jedem Benutzer Profile angelegt, die dessen Präferenzen widerspiegeln. Die Präferenzen können explizit abgefragt oder implizit über die Nutzung von Objekten ermittelt werden. Ziel ist es, einem Nutzer Objekte vorzuschlagen, die zu seinem Benutzerprofil passen (Pazzani und Billsus 2007, S. 325-341). Nach Pazzani und Billsus ist hierfür die Voraussetzung, dass die Objekte im System mit textuellen Attributen versehen sind. So liefern Nachrichten oder Dokumente diese Information im Text, während für Filme beispielsweise Datenbanken mit META-Informationen existieren. Sind solche Attribute nicht vorhanden, können sie zum anderen im Nachhinein ergänzt werden, entweder von Experten oder von Nutzern, beispielsweise durch Tagging der Objekte. Diese Methode ist allerdings sehr aufwändig (Shardanand und Maes 1995, S. 216).

\subsection{Collaborative Filtering}

Der Begriff des Collaborative Filtering wurde von Goldberg et al. (1992) geprägt. Das Konzept zählt derzeit zu den erfolgreichsten Recommender-Systemen und findet sich in den unterschiedlichsten Einsatzgebieten wieder. Das Konzept basiert auf der Einbeziehung der Bewertung anderer Nutzer für ein Objekt. Zunächst wird für den aktiven Nutzer eine Nachbarschaft von ähnlichen Nutzern berechnet. Dies geschieht durch den Vergleich von Nutzungsattributen oder Präferenzangaben. Diese Nachbarschaft bildet die Grundlage für die Erstellung einer Empfehlungsliste für den aktiven Nutzer. Pro Nutzer wird hierbei eine Liste von Bewertungen für besuchte Objekte erstellt. Die Bewertung kann dabei explizit durch Befragung oder implizit durch die Analyse von Objektaufrufen erfolgen. Somit kann für Objekte, die ein Nutzer noch nicht besucht hat, aus der Summe der Bewertungen seiner Nachbarschaft ein Prognosewert berechnet werden. Dieser soll die Relevanz des Objektes für den Nutzer widerspiegeln (Breese et al. 1998, S. 44).

\subsection{Collaborative versus Content-Based Filtering}

Das Content-Based Filtering eignet sich besonders für Objekte die textueller Natur sind oder sich leicht mit Schlüsselwörtern versehen lassen. Die Transparenz des Verfahrens führt dazu, dass es kaum Überraschungseffekte gibt, da der Algorithmus nur Inhalte mit Präferenzen vergleicht (Schafer et al. 2007, S. 300; Adomavicius und Tuzhilin 2005, S. 737). Das Collaborative Filtering Verfahren ist dagegen unabhängig von Objektbeschreibungen und bei Verwendung impliziter Bewertungen unabhängig von Nutzereingaben. Allerdings ist das Verfahren weniger transparent für den Anwender und setzt voraus, dass alle Objekte durch Be- 
wertungen abgedeckt sein müssen - siehe auch „New-Item“-Problem bei Adomavicius und Tuzhilin (2005, S. 740).

Aufgrund zweier Argumente wird im Collaborative Filterting das bessere Verfahren für ein Recommender System in einem Unternehmensportal gesehen: Zum einen bietet das Portal im hier betrachteten Kontext Dienste an, die im Gegensatz zu Wissensmanagement-Diensten oft keine textuellen Inbalte anbieten. Deren Funktionalität müsste somit zunächst detailliert beschrieben werden. Zum anderen müsste sichergestellt werden, dass ein einheitliches Vokabular für die Beschreibungen gefunden wird. Der Einsatz von Unternehmensportalen bietet sich jedoch insbesondere dann an, wenn die Mitarbeiter geographisch und organisatorisch verteilt sind. In diesem Fall kann jedoch nicht von einem gemeinschaftlichen Vokabular auszugegangen werden, da die gleichen Dienste womöglich von verschiedenen Abteilungen für verschiedene Prozesse genutzt werden. Um alle Sichtweisen abbilden zu können, müsste, wie bereits im ersten Punkt kritisiert, mit hohem Aufwand eine Ontologie eingeführt und gepflegt werden. Für das Collaborative Filtering Verfahren liefert ein Unternehmensportal hingegen gute Voraussetzungen. Da alle Nutzer innerhalb des Unternehmensnetzes authentifiziert sind, kennt das System den Nutzer im Idealfall mit erweiterten Profilangaben wie Position und Abteilung. Über diese Daten können schnell verwandte Nutzergruppen identifiziert werden. Zudem gibt jeder Nutzer über die regelmäßige Nutzung einzelner Dienste eine klare Bewertung ab. In der einfachsten Form kann dies wie folgt interpretiert werden: Wird ein Dienst nur wenige Mal besucht und danach nie wieder, so stellt dies eine negative Bewertung dar. Wird ein Dienst regelmäßig genutzt, so wird dies positive bewertet. Ein Collaborative Filtering Recommender-System könnte somit funktionieren, ohne dass die Nutzer explizit Bewertungen abgegeben müssen.

\subsection{Model-Based versus Memory-Based Algorithmen}

Breese et al. (1998, S. 44-49) teilen Algorithmen für Collaborative Filtering Systeme in zwei Kategorien ein: Model-Based und Memory-Based. Für Model-Based Algorithmen wird ein Modell erstellt, das als Grundlage für den Empfehlungsprozess dient. Dieses Modell wird offline aus historischen Daten berechnet und trainiert. In einem zweiten Schritt werden anhand dieses Modells Empfehlungen für jeden Nutzer berechnet. Bekannte Beispiele solcher Algorithmen sind das ClusterVerfahren, das Bayesian Network Model (Breese et al 1998, S. 48) oder das Dependency Network (Calderón-Benavides et al. 2004, S. 1038). Vorteile dieses Ansatzes liegen in der Skalierbarkeit, wobei dieser eine schlechte Prognosegüte gegenüber steht. Da das Modell offline trainiert wird ist es zudem schwer, auf aktuelle Information zu reagieren.

Memory-Based Algorithmen arbeiten im Gegensatz dazu auf der kompletten Datenmatrix. Die Relevanz eines noch nicht bewerteten Objekts wird pro Nutzer auf Grundlage der Bewertung von verwandten Nutzern berechnet. Verglichen mit Model-Based Algorithmen ist der Aufbau grundsätzlich einfacher. Weiterhin kön- 
nen neue Daten einfach hinzugefügt werden, wodurch das System schnell auf Änderungen reagieren kann. Nachteile liegen primär bei der Skalierbarkeit (Deshpande und Karypis 2004, S. 145). Bekannte Algorithmen dieser Klasse sind Pearson-Korrelation (Herlocker et al. 2004, S. 30) und das VektorÄhnlichkeitsmaß (Breese et al. 1998, S. 45).

In der Literatur werden einige empirische Analysen verschiedener Ansätze beschrieben. Sowohl die Analyse von Breese et al. als auch die von CalderonBenavides et al. (2004, S. 1038-1039) kommen dabei zu dem Schluss, dass Memory-Based Algorithmen ihre Stärke vor allem bei wenigen vorhandenen Bewertungen haben, wohingegen Model-Based Algorithmen in diesem Fall einen hohen Informationsverlust verursachen. Im hier beschriebenen Kontext sind eher wenige Bewertungen in Form von Nutzungszahlen vorhanden. Dafür existieren aber genauere Profilangaben für jeden Nutzer, über die sich Nachbarschaften bilden lassen. Da die Zahl der Nutzer und Objekte klein ist kann die Skalierbarkeit des Algorithmus vernachlässigt werden. Im Vordergrund steht das Ziel „find all good items". Aus diesen Gründen erscheinen uns Memory-Based Algorithmen als geeignete Variante für Unternehmensportale.

\subsection{Weitere Aspekte für das Design des Recommender Systems}

Bei der weiteren Gestaltung eines solchen Systems müssen auch auf den konkreten Unternehmenskontext speziell abgestimmte Design-Entscheidungen getroffen werden, deren Vor- oder Nachteilhaftigkeit sich nur durch Simulationen oder Testläufe mit den tatsächlichen Nutzungsdaten und Dienstgruppen des im Einsatz befindlichen Portals herausarbeiten lässt.

Berechnung der Ähnlichkeiten zwischen den Nutzern: Für jeden Nutzer ist bekannt, welche Dienste er regelmäßig nutzt. Diese Information kann verwendet werden, um ähnliche Nutzer anhand ihres Nutzungsprofils zu identifizieren. Diese Berechnung kann nach Breese unter Verwendung verschiedenen Algorithmen erfolgen: der Berechnung eines angepassten Korrelationskoeffizienten, der Vector Similarity, der Inverse User Frequency oder durch default voting (1998, S. 45ff). Vergleichende Tests zu diesen Ansätzen arbeiten jedoch mit wesentlich größeren Datensätzen. Hier gilt es zu testen, welcher Algorithmus für kleinere Datensätze am besten geeignet ist. So ist die Verwendung des Korrelationskoeffizienten nach Resnick (1994, S. 181) beispielsweise kritisch zu bewerten, da pro Nutzer zu wenige unterschiedliche Bewertungen vorliegen und die Korrelation daher nicht hinlänglich berechnet werden kann.

Implizite Berechnung des Ratings: Für jeden Nutzer werden anhand der besuchten Dienste implizit Ratings für diese angenommen. Wie diese Umwandlung abläuft muss je nach Art der angebotenen Dienste entschieden werden. So kann es beispielsweise vorkommen, dass sich die Dienste derart unterscheiden, dass einige mehrfach täglich sinnvoll genutzt werden können, während andere Dienste nur unregelmäßig oder in großen zeitlichen Abständen (z. B. zum Quartalsabschluss) 
genutzt werden können. Diese Saisonalität und Heterogenität der Dienste, die typisch für Unternehmensportale sind, müssen bei der Berechnung der Ratings berücksichtig werden. Dies kann beispielsweise über eine unterschiedliche Gewichtung der jeweiligen Dienstbewertung oder durch unterschiedlich große Erfassungszeiträume, über die die Bewertung normiert wird, reguliert werden.

Zabl der angezeigten Empfeblungen: Bei der Wahl der Anzeige von Empfehlungen muss zwischen dem Top-N Ansatz und der Anzeige aller über einem schwellenwert liegenden Empfehlungen gewählt werden (Herlocker et al. 2004, S. 24). Die Entscheidung wird davon abhängen, wie viele Empfehlungen bei jedem dieser Ansätze pro Nutzer angezeigt würden. Dies kann nur durch Tests auf bestehenden Daten erfolgen. Zu viele angezeigte Empfehlungen würden den Nutzer eher davon abhalten, sich diese anzuschauen.

Sicherbeitsaspekte: Im Unternehmenskontext kann es auch vorkommen, dass prinzipiell Dienste empfohlen werden, für die der jeweilige Nutzer keine Berechtigung hat. Es muss daher entschieden werden, ob diese Empfehlungen unterdrückt werden, oder ob eine Beschreibung des Dienstes angezeigt wird, so dass der Nutzer gegebenenfalls eine Freigabe beantragen kann. Diese Eigenschaft könnte weiter gehend auch genutzt werden, um beim Anlegen neuer Nutzer basierend auf deren Profildaten und den Ergebnissen des Recommender-Systems von Beginn an passende Dienste freizuschalten.

Anzeige der Empfehlungen: Nicht zuletzt gilt es auch zu untersuchen, wie die Empfehlungen angezeigt werden können, so dass sie den Arbeitsfluss des Mitarbeiters nicht behindern aber trotzdem wahrgenommen werden. Das Einblenden von Pop-ups würde sicherlich die Sichtbarkeit des Systems sicherstellen, allerdings höchstwahrscheinlich auch zu zahlreichen Beschwerden führen. Eine schlichte Anzeige der Empfehlungen beispielsweise in einer Spalte auf der Seite kann hingegen dazu führen, dass sie gar nicht wahrgenommen werden. Auch hier wird es sich empfehlen, durch Usability-Tests festzustellen, welche Art der Anzeige am besten geeignet ist.

\subsection{Ausblick}

Die Evolution von Unternehmensportalen hin zu komplexen Enterprise Application Suites birgt große Potentiale für Produktivitätssteigerungen, setzt aber auch voraus, dass diese durch die Mitarbeiter ausgenutzt werden können. Das Portalmanagement muss daher insbesondere dafür sorgen, dass die Mitarbeiter effektiv in dem Auffinden individuell relevanter Dienste unterstützt werden. Zu diesem Zweck wird ein Recommender-System vorgeschlagen, welches auf dem Collaborative Filtering Ansatz unter der Verwendung von Memory-Based Algorithmen aufsetzt. Für den konkreten Einsatz im Unternehmen sind jedoch weitere DesignEntscheidungen auf Grundlage des vorliegenden Nutzungsumfeldes und der Datenbasis zu treffen. Ebenso kann es arbeitsrechtliche Bedenken geben, da für die Berechnung der Empfehlungen Nutzerinformationen erhoben und ausgewertet 
werden müssen. Eine Einschränkung oder Anonymisierung bei der Datenerhebung hätte jedoch unweigerlich auch Auswirkungen auf die Prognosegüte des Recommender-Systems.

\section{Literatur}

Adomavicius G, Tuzhilin A (2005) Toward the next generation of recommender systems: a survey of the state-of-the-art and possible extensions. IEEE Transactions on Knowledge and Data Engineering 17(6): 734-749.

Aneja A, Rowan C, Brooksby B (2000) Corporate portal framework for transforming content chaos on intranets. Intel Technology Journal 4(1).

Arbin K, Essler U (2002) Emerging Industrial eMarkets: The case of Covisint in Europe. In: Proceedings of the 15th International Electronic Commerce Conference: 279-292.

Benbya H, Passiante G, Belbaly N (2004) Corporate portal: a tool for knowledge management synchronization. International Journal of Information Management 24(3): 201-220.

Breese J, Heckerman D, Kadie C (1998) Empirical analysis of predictive algorithms for collaborative filtering. In: Proceedings of the 14th Annual Conference on Uncertainty in Artificial Intelligence (UAI-98): 43-52.

Calderón-Benavides M, González-Caro C, Pérez-Alcázar J, Garciaz J, Delgado J (2004) A comparison of several predictive algorithms for collaborative filtering on multi-valued ratings. In: Proceedings of the 2004 ACM symposium on Applied computing: 1033-1039.

Deshpande M, Karypis G (2004) Item-based top-N recommendation algorithms. ACM Transactions on Information Systems 22(1): 143-177.

Detlor B (2000) The corporate portal as information infrastructure: towards a framework for portal design. International Journal of Information Management 20(2): 91-101.

Dias C (2001) Corporate portals: a literature review of a new concept in Information Management. International Journal of Information Management 21(4): 269-287.

Eckerson, W (1999) 15 rules for enterprise portals. Oracle Magazine 13(4): 13-14.

Elsner H, Vo K (2007) Management of Portal Evolution - Introducing evolution management for the Corporate Financial Portal. In Wirtschaftsinformatik Proceedings: 337-352. 
Goldberg D, Nichols D, Oki B, Terry D (1992) Using collaborative filtering to weave an information tapestry. Communications of the ACM 35(12): 61-70.

Herlocker J, Konstan J, Terveen L, Riedl J (2004) Evaluating collaborative filtering recommender systems. ACM Trans. on Inf. Sys. 22(1): 5-53.

Knorr E (2004) The new enterprise portal: The browser-based portal is fast becoming the enterprise UI and the nexus for a new breed of integration and app dev. http://www.infoworld.com/article/04/01/09/02FEportal_1.html

Montaner M, López B, de La Rosa J (2003) A Taxonomy of Recommender Agents on the Internet. In: Artificial Intelligence Review 19(4): 285-330.

Murray G (1999) The portal is the desktop.

Pazzani M, Billsus D (2007) Content-based recommendation systems. The adaptive web: Methods and strategies of web personalization. Springer Heidelberg: 325-341.

Resnick P, Iacovou N, Suchak M, Bergstrom P, Riedl J (1994) GroupLens: an open architecture for collaborative filtering of Netnews. In: CSCW Proceedings: $175-186$.

Schafer J, Frankowski D, Herlocker J, Sen S (2007) Collaborative filtering recommender systems. In: The Adaptive Web: Methods and Strategies of Web Personalization, Springer: 291-324.

Shardanand U, Maes P (1995) Social information filtering: algorithms for automating "word of mouth". In: Proceedings of the ACM Conference on Human Factors in Computing Systems (CHI'95): 210-217.

Shilakes C, Tylman J (1998) Enterprise information portals. Merrill Lynch In-depth report.

Terra J, Gordon C (2003) Realizing the promise of corporate portals: Leveraging knowledge for business success. Butterworth-Heinemann, Amsterdam.

White M (2000) Enterprise information portals. The Electronic Library 18(5): 354 362.

White C (2003) Is the portal dead? Information Management Magazine: 1-3.

Zanker M, Aschinger M, Jessenitschnig M (2007) Development of a collaborative and constraint-based web configuration system for personalized bundling of products and services. Web Information Systems Engineering: 273-284. 